FTUV/97-47

IFIC/97-63

UG-FT-77/97

hep-ph/9804462

April 1998

\title{
PERTURBATIVE QUARK MASS CORRECTIONS TO THE TAU HADRONIC WIDTH
}

\author{
Antonio Pich ${ }^{a}$ and Joaquim Prades ${ }^{b}$ \\ ${ }^{a}$ Departament de Física Teòrica, IFIC, Universitat de València - CSIC \\ Dr. Moliner 50, E-46100 Burjassot (València), Spain. \\ ${ }^{b}$ Departamento de Física Teórica y del Cosmos, Universidad de Granada, \\ Campus de Fuente Nueva, E-18002 Granada, Spain.
}

\begin{abstract}
The perturbative quark-mass corrections to the $\tau$ hadronic width are analysed to $O\left(\alpha_{s}^{3} m_{q}^{2}\right)$, using the presently available theoretical information. The behaviour of the perturbative series is investigated in order to assess the associated uncertainties. The implications for the determination of the strange quark mass from $\tau$ decay data are discussed.
\end{abstract}




\section{Introduction}

The inclusive character of the total $\tau$ hadronic width renders possible an accurate calculation of the ratio [1-5]

$$
R_{\tau} \equiv \frac{\Gamma\left[\tau^{-} \rightarrow \nu_{\tau} \text { hadrons }(\gamma)\right]}{\Gamma\left[\tau^{-} \rightarrow \nu_{\tau} e^{-} \bar{\nu}_{e}(\gamma)\right]},
$$

using standard field theory methods. The result turns out to be very sensitive to the value of $\alpha_{s}\left(M_{\tau}^{2}\right)$. Moreover, the uncertainties in the theoretical calculation are quite small and dominated by the perturbative errors. This has been used to perform a very precise determination of the QCD coupling at low energies [5].

Quark masses play a rather minor rôle in $R_{\tau}$. Owing to the tiny values of $m_{u}$ and $m_{d}$, their associated corrections are very small [3] $(\sim-0.1 \%)$. The strange quark contribution to the total $\tau$ hadronic width is suppressed by the Cabibbo factor $\left|V_{u s}\right|^{2}$, which puts the induced $m_{s}$ correction also at the per cent level. However, if one analyses separately the semi-inclusive decay width of the $\tau$ into Cabibbo-suppressed modes (i.e. final states with an odd number of kaons), the relatively large value of $m_{s}$ induces an important effect of a size similar to the massless perturbative correction and of opposite sign [3]. The corresponding $R_{\tau, S}$ prediction is then very sensitive to the strange quark mass and could be used to extract information on this important, and nowadays controversial, parameter. A very preliminary value of $m_{s}$, extracted from the ALEPH $\tau$ decay data, has been already presented in recent workshops [6,7].

The determinations of light quark masses are usually obtained from analyses of the divergences of the vector and axial-vector current two-point function correlators or related observables [8 13]. These correlators are proportional to quark masses and, therefore, are very sensitive to their numerical values. Unfortunately, one needs phenomenological information on the associated scalar and pseudo-scalar spectral functions, which are not well known at present. The obvious advantage of a possible determination of $m_{s}$ analysing quark mass effects in $\tau$ decays is that the experimental error can be systematically reduced in foreseen facilities like tau-charm or B factories. There is then some hope to achieve a precise determination of $m_{s}$ from such analyses.

Recently the $O\left(\alpha_{s}^{3}\right)$ corrections to the $J=0$ quark correlators have been calculated [14], and have been found to be rather large. The influence of these $O\left(\alpha_{s}^{3}\right)$ corrections on the determination of quark masses and the uncertainties coming from the truncation of the QCD perturbative series depend very much on the observable. One can see for instance that the QCD perturbative series behaves geometrically to $O\left(\alpha_{s}^{3}\right)$ for the divergence of pseudo-scalar (scalar) currents if

resummed perturbatively in terms of $\alpha_{s}(s)$ [12,13]. This convergence improves [13] using other resummations like the Principle of Minimal Sensitivity (PMS) [15] or the one advocated in Ref. [4]. 
The hadronic $\tau$ decay width has also a $J=0$ contribution, which, as we shall see, behaves rather badly. However, the largest quark-mass correction originates in a piece of the left-handed current correlation function, involving the $J=0+1$ combination, which shows a much better perturbative convergence.

The purpose of this paper is to study the perturbative behaviour of the corrections to $R_{\tau}$ which are proportional to $m_{q}^{2}$, in order to assess the associated uncertainties. These are the leading theoretical uncertainties in the $m_{s}$ determination. The $O\left(\alpha_{s} m_{q}^{2}\right)$ contributions were already studied in Ref. [3]. In Ref. [16] the contributions of $O\left(\alpha_{s}^{2} m_{q}^{2}\right)$ to the relevant correlators were worked out. More recently, some partial information on $O\left(\alpha_{s}^{3} m_{q}^{2}\right)$ corrections has become available [14, 17]. A much detailed analysis of all contributions up to dimension four will be presented elsewhere.

\section{Theoretical Framework}

The theoretical analysis of $R_{\tau}$ involves the two-point correlation functions for the vector $V_{i j}^{\mu}=\bar{\psi}_{j} \gamma^{\mu} \psi_{i}$ and axial-vector $A_{i j}^{\mu}=\bar{\psi}_{j} \gamma^{\mu} \gamma_{5} \psi_{i}$ colour-singlet quark currents $(i, j=u, d, s)$ :

$$
\begin{aligned}
\Pi_{i j, V}^{\mu \nu}(q) & \equiv i \int d^{4} x e^{i q x}\left\langle 0\left|T\left(V_{i j}^{\mu}(x) V_{i j}^{\nu}(0)^{\dagger}\right)\right| 0\right\rangle, \\
\Pi_{i j, A}^{\mu \nu}(q) & \equiv i \int d^{4} x e^{i q x}\left\langle 0\left|T\left(A_{i j}^{\mu}(x) A_{i j}^{\nu}(0)^{\dagger}\right)\right| 0\right\rangle .
\end{aligned}
$$

They have the Lorentz decompositions

$$
\Pi_{i j, V / A}^{\mu \nu}(q)=\left(-g^{\mu \nu} q^{2}+q^{\mu} q^{\nu}\right) \Pi_{i j, V / A}^{(1)}\left(q^{2}\right)+q^{\mu} q^{\nu} \Pi_{i j, V / A}^{(0)}\left(q^{2}\right),
$$

where the superscript $(J)$ in the transverse and longitudinal components denotes the corresponding angular momentum $J=1(\mathrm{~T})$ and $J=0(\mathrm{~L})$ in the hadronic rest frame.

The imaginary parts of the two-point functions $\Pi_{i j, V / A}^{(J)}\left(q^{2}\right)$ are proportional to the spectral functions for hadrons with the corresponding quantum numbers. The semi-hadronic decay rate of the $\tau$ can be written as an integral of these spectral functions over the invariant mass $s$ of the final-state hadrons:

$$
R_{\tau}=12 \pi \int_{0}^{M_{\tau}^{2}} \frac{d s}{M_{\tau}^{2}}\left(1-\frac{s}{M_{\tau}^{2}}\right)^{2}\left[\left(1+2 \frac{s}{M_{\tau}^{2}}\right) \operatorname{Im} \Pi^{(1)}(s)+\operatorname{Im} \Pi^{(0)}(s)\right] .
$$

The appropriate combinations of correlators are

$$
\Pi^{(J)}(s) \equiv\left|V_{u d}\right|^{2}\left(\Pi_{u d, V}^{(J)}(s)+\Pi_{u d, A}^{(J)}(s)\right)+\left|V_{u s}\right|^{2}\left(\Pi_{u s, V}^{(J)}(s)+\Pi_{u s, A}^{(J)}(s)\right) .
$$

We can decompose the predictions for $R_{\tau}$ into contributions associated with specific quark currents:

$$
R_{\tau}=R_{\tau, V}+R_{\tau, A}+R_{\tau, S}
$$


$R_{\tau, V}$ and $R_{\tau, A}$ correspond to the contributions from the first two terms in Eq. (6), while $R_{\tau, S}$ contains the remaining Cabibbo-suppressed contributions.

Exploiting the analytic properties of the correlators $\Pi^{(J)}(s)$, Eq. (5) can be expressed as a contour integral in the complex $s$ plane running counter-clockwise around the circle $|s|=M_{\tau}^{2}$ :

$$
R_{\tau}=-\pi i \oint_{|s|=M_{\tau}^{2}} \frac{d s}{s}\left(1-\frac{s}{M_{\tau}^{2}}\right)^{3}\left\{3\left(1+\frac{s}{M_{\tau}^{2}}\right) D^{L+T}(s)+4 D^{L}(s)\right\} .
$$

We have used integration by parts to rewrite $R_{\tau}$ in terms of the logarithmic derivative of the relevant correlators,

$$
D^{L+T}(s) \equiv-s \frac{d}{d s}\left[\Pi^{(0+1)}(s)\right], \quad D^{L}(s) \equiv \frac{s}{M_{\tau}^{2}} \frac{d}{d s}\left[s \Pi^{(0)}(s)\right],
$$

which satisfy homogeneous renormalization group equations.

Using the Operator Product Expansion to organise the perturbative and nonperturbative contributions to the correlators into a systematic expansion [18 in powers of $1 / s$, the total ratio $R_{\tau}$ can be expressed as an expansion in powers of $1 / M_{\tau}^{2}$, with coefficients that depend only logarithmically on $M_{\tau}$ [3]:

$R_{\tau}=3\left(\left|V_{u d}\right|^{2}+\left|V_{u s}\right|^{2}\right) S_{E W}\left\{1+\delta_{E W}^{\prime}+\delta^{(0)}+\sum_{D=2,4, \ldots}\left(\cos ^{2} \theta_{C} \delta_{u d}^{(D)}+\sin ^{2} \theta_{C} \delta_{u s}^{(D)}\right)\right\}$,

where $\delta_{i j}^{(D)}=\left(\delta_{i j, V}^{(D)}+\delta_{i j, A}^{(D)}\right) / 2$ is the average of the vector and axial-vector corrections of dimension $D, S_{E W}$ and $\delta_{E W}^{\prime}$ contain the known 19, 20 electroweak corrections, and $\sin ^{2} \theta_{C} \equiv\left|V_{u s}\right|^{2} /\left(\left|V_{u d}\right|^{2}+\left|V_{u s}\right|^{2}\right)$.

The dimension-zero contribution is the purely perturbative correction neglecting quark masses, which, owing to chiral symmetry, is identical for the vector and axial-vector correlators. It is fully generated by the Adler function $D^{L+T}(s)$, because $D^{L}(s)$ vanishes in the chiral limit. The correction $\delta^{(0)}$ has been investigated in great detail in Ref. [4]. We will follow a similar procedure to analyse the perturbative quark-mass corrections of dimension two.

\section{Dimension-Two Corrections}

For the sake of simplicity, let us take here $m_{u}=m_{d}=0$. The arguments we shall put forward don't depend on it. In this limit, the vector and axial-vector correlators get the same quark-mass corrections, i.e. $D_{u s, A}^{J}(s)=D_{u s, V}^{J}(s) \equiv$ $D_{u s}^{J}(s) \quad(J=L+T, L)$. The dimension-two contributions can be written in the form:

$$
\begin{aligned}
\left.D_{u s}^{L+T}(s)\right|_{D=2} & =\frac{3}{4 \pi^{2}} \frac{m_{s}^{2}\left(-\xi^{2} s\right)}{s} \sum_{n=0} \tilde{d}_{n}^{L+T}(\xi) a^{n}\left(-\xi^{2} s\right), \\
\left.D_{u s}^{L}(s)\right|_{D=2} & =-\frac{3}{8 \pi^{2}} \frac{m_{s}^{2}\left(-\xi^{2} s\right)}{M_{\tau}^{2}} \sum_{n=0} \tilde{d}_{n}^{L}(\xi) a^{n}\left(-\xi^{2} s\right),
\end{aligned}
$$


where $a=\alpha_{s} / \pi, \xi$ is an arbitrary scale factor (of order unity) and the coefficients $\tilde{d}_{n}^{J}(\xi)$ are constrained by the homogeneous renormalization group equations satisfied by the corresponding functions $D_{u s}^{J}(s)$ :

$$
\xi \frac{d}{d \xi} \tilde{d}_{n}^{J}(\xi)=\sum_{k=1}^{n}\left[2 \gamma_{k}-(n-k) \beta_{k}\right] \tilde{d}_{n-k}^{J}(\xi),
$$

for $n \geq 1$ and

$$
\frac{d}{d \xi} \tilde{d}_{0}^{J}(\xi)=0
$$

i.e.

$$
\begin{aligned}
\tilde{d}_{0}^{J}(\xi)= & d_{0}^{J} \\
\tilde{d}_{1}^{J}(\xi)= & d_{1}^{J}+2 \gamma_{1} d_{0}^{J} \log \xi \\
\tilde{d}_{2}^{J}(\xi)= & d_{2}^{J}+\left[2 \gamma_{2} d_{0}^{J}+\left(2 \gamma_{1}-\beta_{1}\right) d_{1}^{J}\right] \log \xi+\gamma_{1}\left(2 \gamma_{1}-\beta_{1}\right) d_{0}^{J} \log ^{2} \xi, \\
\tilde{d}_{3}^{J}(\xi)= & d_{3}^{J}+\left[2 \gamma_{3} d_{0}^{J}+\left(2 \gamma_{2}-\beta_{2}\right) d_{1}^{J}+2\left(\gamma_{1}-\beta_{1}\right) d_{2}^{J}\right] \log \xi \\
& +\left[\left(-\gamma_{1} \beta_{2}+2 \gamma_{2}\left(2 \gamma_{1}-\beta_{1}\right)\right) d_{0}^{J}+\left(\gamma_{1}-\beta_{1}\right)\left(2 \gamma_{1}-\beta_{1}\right) d_{1}^{J}\right] \log ^{2} \xi \\
& +\frac{2}{3} \gamma_{1}\left(\gamma_{1}-\beta_{1}\right)\left(2 \gamma_{1}-\beta_{1}\right) d_{0}^{J} \log ^{3} \xi \\
\tilde{d}_{4}^{J}(\xi)= & d_{4}^{J}+\cdots
\end{aligned}
$$

The factors $\beta_{k}$ and $\gamma_{k}$ are the expansion coefficients of the QCD $\beta$ and $\gamma$ functions,

$$
\begin{aligned}
\mu \frac{d a}{d \mu} & =\beta(a) a, & \beta(a) & =\sum_{k=1} \beta_{k} a^{k}, \\
\mu \frac{d m}{d \mu} & =-\gamma(a) m, & \gamma(a) & =\sum_{k=1} \gamma_{k} a^{k},
\end{aligned}
$$

which are known to four loops 21 23. The coefficients $d_{n}^{J} \equiv \tilde{d}_{n}^{J}(1)$ are only known to order $\alpha_{s}^{2}$ for $J=L+T$ and $\alpha_{s}^{3}$ for $J=L$ [8, 14, 17, 24, 28].

For three flavours and in the $\overline{\mathrm{MS}}$ scheme, one has:

$$
\begin{gathered}
\beta_{1}=-\frac{9}{2} ; \quad \beta_{2}=-8 ; \quad \beta_{3}=-\frac{3863}{192} ; \\
\beta_{4}=-\frac{140599}{2304}-\frac{445}{16} \zeta_{3} \approx-94.456079 \\
\gamma_{1}=2 ; \quad \gamma_{2}=\frac{91}{12} ; \quad \gamma_{3}=\frac{8885}{288}-5 \zeta_{3} \approx 24.840410 \\
\gamma_{4}=\frac{2977517}{20736}-\frac{9295}{216} \zeta_{3}+\frac{135}{8} \zeta_{4}-\frac{125}{6} \zeta_{5} \approx 88.525817
\end{gathered}
$$




$$
\begin{gathered}
d_{0}^{L+T}=1 ; \quad d_{1}^{L+T}=\frac{13}{3} ; \quad d_{2}^{L+T}=\frac{21541}{432}+\frac{323}{54} \zeta_{3}-\frac{520}{27} \zeta_{5} \approx 37.083047 ; \\
d_{0}^{L}=1 ; \quad d_{1}^{L}=\frac{17}{3} ; \quad d_{2}^{L}=\frac{9631}{144}-\frac{35}{2} \zeta_{3} \approx 45.845949 ; \\
d_{3}^{L}=\frac{4748953}{5184}-\frac{91519}{216} \zeta_{3}-\frac{5}{2} \zeta_{4}+\frac{715}{12} \zeta_{5} \approx 465.846304
\end{gathered}
$$

Notice the rather bad perturbative behaviour of the $D=2$ corrections to the correlation functions $D_{u s}^{J}$. Remember that $a\left(M_{\tau}^{2}\right) \simeq 0.11$.

Inserting the expansions (11) and (12) in Eq. (8), the $D=2$ corrections to $R_{\tau, S}$ can be expressed as

$$
\delta_{u s}^{(2)}=-8 \frac{m_{s}^{2}\left(M_{\tau}^{2}\right)}{M_{\tau}^{2}} \Delta\left[a\left(M_{\tau}^{2}\right)\right], \quad \Delta[a] \equiv \frac{1}{4}\left\{3 \Delta^{L+T}[a]+\Delta^{L}[a]\right\},
$$

where

$$
\Delta^{J}\left[a\left(M_{\tau}^{2}\right)\right]=\sum_{n=0} \tilde{d}_{n}^{J}(\xi) B_{J}^{(n)}\left(a_{\xi}\right)
$$

and the contour integrations are contained in the functions

$$
\begin{aligned}
B_{L+T}^{(n)}\left(a_{\xi}\right) & \equiv \frac{-1}{4 \pi i} \oint_{|x|=1} \frac{d x}{x^{2}}(1+x)(1-x)^{3}\left(\frac{m\left(-\xi^{2} M_{\tau}^{2} x\right)}{m\left(M_{\tau}^{2}\right)}\right)^{2} a^{n}\left(-\xi^{2} M_{\tau}^{2} x\right), \\
B_{L}^{(n)}\left(a_{\xi}\right) & \equiv \frac{1}{2 \pi i} \oint_{|x|=1} \frac{d x}{x}(1-x)^{3}\left(\frac{m\left(-\xi^{2} M_{\tau}^{2} x\right)}{m\left(M_{\tau}^{2}\right)}\right)^{2} a^{n}\left(-\xi^{2} M_{\tau}^{2} x\right) .
\end{aligned}
$$

Since the quark mass ratio is flavour independent, the integrals $B_{J}^{(n)}\left(a_{\xi}\right)$ regulate also the small corrections proportional to $m_{u}$ and $m_{d}$, which we are neglecting. These functions depend only on $\beta_{i}, \gamma_{j}, a_{\xi} \equiv \alpha_{s}\left(\xi^{2} M_{\tau}^{2}\right) / \pi$ and $\log \xi$. Moreover, they satisfy the following homogeneous renormalization group equations:

$$
\xi \frac{d}{d \xi} B_{J}^{(n)}\left(a_{\xi}\right)=\sum_{k=1}\left(n \beta_{k}-2 \gamma_{k}\right) B_{J}^{(n+k)}\left(a_{\xi}\right) .
$$

\section{Perturbative $a_{\xi}$ Expansion}

The usual perturbative approach expands the $B_{J}^{(n)}\left(a_{\xi}\right)$ functions in powers of $a_{\xi}$. This gives,

$$
\begin{aligned}
B_{J}^{(0)}\left(a_{\xi}\right) & =1-\gamma_{1}\left[2 \log \xi+H_{1}^{J}\right] a_{\xi} \\
- & {\left[2 \gamma_{2} \log \xi-\gamma_{1}\left(2 \gamma_{1}+\beta_{1}\right) \log ^{2} \xi+\left(\gamma_{2}-2 \gamma_{1}^{2} \log \xi\right) H_{1}^{J}\right.} \\
& \left.-\frac{\gamma_{1}}{2}\left(\gamma_{1}-\frac{\beta_{1}}{2}\right) H_{2}^{J}\right] a_{\xi}^{2}
\end{aligned}
$$




$$
\begin{aligned}
- & {\left[2 \gamma_{3} \log \xi-\left(2 \gamma_{2}\left(2 \gamma_{1}+\beta_{1}\right)+\gamma_{1} \beta_{2}\right) \log ^{2} \xi+\frac{2}{3} \gamma_{1}\left(\gamma_{1}+\beta_{1}\right)\left(2 \gamma_{1}+\beta_{1}\right) \log ^{3} \xi\right.} \\
& +\left(\gamma_{3}-4 \gamma_{1} \gamma_{2} \log \xi+\gamma_{1}^{2}\left(2 \gamma_{1}+\beta_{1}\right) \log ^{2} \xi\right) H_{1}^{J} \\
& +\left(\frac{1}{2} \beta_{1} \gamma_{2}+\frac{1}{4} \beta_{2} \gamma_{1}-\gamma_{1} \gamma_{2}+\gamma_{1}^{2}\left(\gamma_{1}-\frac{\beta_{1}}{2}\right) \log \xi\right) H_{2}^{J} \\
& \left.+\frac{\gamma_{1}}{12}\left(2 \gamma_{1}-\beta_{1}\right)\left(\gamma_{1}-\beta_{1}\right) H_{3}^{J}\right] a_{\xi}^{3}+\cdots \\
B_{J}^{(1)}\left(a_{\xi}\right) & =a_{\xi}-\left[2 \gamma_{1} \log \xi+\left(\gamma_{1}-\frac{\beta_{1}}{2}\right) H_{1}^{J}\right] a_{\xi}^{2} \\
- & {\left[2 \gamma_{2} \log (\xi)-\gamma_{1}\left(2 \gamma_{1}+\beta_{1}\right) \log ^{2} \xi+\left(\gamma_{2}-\frac{\beta_{2}}{2}-\gamma_{1}\left(2 \gamma_{1}-\beta_{1}\right) \log \xi\right) H_{1}^{J}\right.} \\
& \left.-\frac{1}{2}\left(\gamma_{1}-\frac{\beta_{1}}{2}\right)\left(\gamma_{1}-\beta_{1}\right) H_{2}^{J}\right] a_{\xi}^{3}+\cdots \\
B_{J}^{(2)}\left(a_{\xi}\right) & =a_{\xi}^{2}-\left[2 \gamma_{1} \log \xi+\left(\gamma_{1}-\beta_{1}\right) H_{1}^{J}\right] a_{\xi}^{3}+\cdots \\
B_{J}^{(3)}\left(a_{\xi}\right) & =a_{\xi}^{3}-\cdots
\end{aligned}
$$

where

$$
\begin{gathered}
H_{n}^{L+T} \equiv \frac{-1}{4 \pi i} \oint_{|x|=1} \frac{d x}{x^{2}}(1+x)(1-x)^{3} \log ^{n}(-x), \\
H_{n}^{L} \equiv \frac{1}{2 \pi i} \oint_{|x|=1} \frac{d x}{x}(1-x)^{3} \log ^{n}(-x) .
\end{gathered}
$$

To $O\left(\alpha_{s}^{4}\right)$, the needed integrals are

$$
\begin{gathered}
H_{0}^{L+T}=1, \quad H_{1}^{L+T}=\frac{1}{6}, \quad H_{2}^{L+T}=\frac{25}{18}-\frac{\pi^{2}}{3}, \\
H_{3}^{L+T}=\frac{85}{36}-\frac{\pi^{2}}{6}, \quad H_{4}^{L+T}=\frac{721}{54}-\frac{25}{9} \pi^{2}+\frac{\pi^{4}}{5}, \\
H_{0}^{L}=1, \quad H_{1}^{L}=-\frac{11}{6}, \quad H_{2}^{L}=\frac{85}{18}-\frac{\pi^{2}}{3}, \\
H_{3}^{L}=-\frac{575}{36}+\frac{11}{6} \pi^{2}, \quad H_{4}^{L}=\frac{3661}{54}-\frac{85}{9} \pi^{2}+\frac{\pi^{4}}{5} .
\end{gathered}
$$

The perturbative expansions $\Delta^{J}[a]$ then take the form

$$
\Delta^{J}\left[a\left(M_{\tau}^{2}\right)\right]=\sum_{n=0}\left[\tilde{d}_{n}^{J}(\xi)+\tilde{h}_{n}^{J}(\xi)\right] a_{\xi}^{n},
$$


where the coefficients $\tilde{h}_{n}^{J}(\xi)$ depend on $\tilde{d}_{m<n}^{J}(\xi), \beta_{m<n}$ and $\gamma_{m \leq n}$; thus, they are known up to $O\left(a^{3}\right)$ and $O\left(a^{4}\right)$ for $J=L+T$ and $J=L$ respectively. For $\xi=1$, one has $\left[h_{n}^{J} \equiv \tilde{h}_{n}^{J}(1)\right]$ :

$$
\begin{gathered}
h_{0}^{L+T}=0 ; \quad h_{1}^{L+T}=-\frac{1}{3} ; \quad h_{2}^{L+T}=\frac{113}{72}-\frac{17}{12} \pi^{2} \approx-12.412495 ; \\
h_{3}^{L+T}=\frac{114517}{2592}-\frac{4391}{144} \pi^{2}-\frac{3659}{648} \zeta_{3}+\frac{1690}{81} \zeta_{5} \approx-241.926329 ; \\
h_{4}^{L+T}=\frac{26864009}{13824}-\frac{3110783}{5184} \pi^{2}-\frac{1073}{1920} \pi^{4}+\frac{3051761}{15552} \zeta_{3}-\frac{123745}{2592} \pi^{2} \zeta_{3} \\
\quad-\frac{171845}{243} \zeta_{5}+\frac{29575}{162} \pi^{2} \zeta_{5}-\frac{35}{24} d_{3}^{L+T} \approx-3229.101787-\frac{35}{24} d_{3}^{L+T} ; \\
h_{0}^{L}=0 ; \quad h_{1}^{L}=\frac{11}{3} ; \quad h_{2}^{L}=\frac{625}{8}-\frac{17}{12} \pi^{2} \approx 64.143060 ; \\
h_{3}^{L}=\frac{1435691}{864}-\frac{7927}{144} \pi^{2}-\frac{5225}{24} \zeta_{3} \approx 856.673579 ; \\
h_{4}^{L}=\frac{693706385}{20736}-\frac{2295071}{1728} \pi^{2}-\frac{10877}{17280} \pi^{4}-\frac{1429525}{144} \zeta_{3}+\frac{5595}{32} \pi^{2} \zeta_{3}+\frac{264275}{288} \zeta_{5} \\
\approx 11377.111254
\end{gathered}
$$

The contour integration generates rather large numerical factors, which show an opposite behaviour for the transverse and longitudinal pieces. In the $\Delta^{L+T}$ expansion the $h_{n}^{L+T}$ contributions cancel to some extent with the original correlationfunction coefficients $d_{n}^{L+T}$,

$$
\begin{aligned}
& \Delta^{L+T}\left[a\left(M_{\tau}^{2}\right)\right]=1+4 a\left(M_{\tau}^{2}\right)+\left(\frac{22219}{432}-\frac{17}{12} \pi^{2}+\frac{323}{54} \zeta_{3}-\frac{520}{27} \zeta_{5}\right) a\left(M_{\tau}^{2}\right)^{2} \\
& \quad+\left(d_{3}^{L+T}+\frac{114517}{2592}-\frac{4391}{144} \pi^{2}-\frac{3659}{648} \zeta_{3}+\frac{1690}{81} \zeta_{5}\right) a\left(M_{\tau}^{2}\right)^{3}+\cdots \\
& \quad=1+4 a\left(M_{\tau}^{2}\right)+24.671 a\left(M_{\tau}^{2}\right)^{2}+\left(d_{3}^{L+T}-241.926\right) a\left(M_{\tau}^{2}\right)^{3}+\cdots(37)
\end{aligned}
$$

However, both $d_{n}^{L}$ and $h_{n}^{L}$ contributions are large and positive, which gives rise to a badly behaved expansion for $\Delta^{L}$ :

$$
\begin{aligned}
& \Delta^{L}\left[a\left(M_{\tau}^{2}\right)\right]=1+\frac{28}{3} a\left(M_{\tau}^{2}\right)+\left(\frac{20881}{144}-\frac{17}{12} \pi^{2}-\frac{35}{2} \zeta_{3}\right) a\left(M_{\tau}^{2}\right)^{2} \\
& \quad+\left(\frac{13363099}{5184}-\frac{7927}{144} \pi^{2}-\frac{\pi^{4}}{36}-\frac{17318}{27} \zeta_{3}+\frac{715}{12} \zeta_{5}\right) a\left(M_{\tau}^{2}\right)^{3}+\cdots \\
& =1+9.333 a\left(M_{\tau}^{2}\right)+109.989 a\left(M_{\tau}^{2}\right)^{2}+1322.520 a\left(M_{\tau}^{2}\right)^{3}+\cdots
\end{aligned}
$$


The bold-guess estimate $d_{4}^{L} \sim d_{3}^{L}\left(d_{3}^{L} / d_{2}^{L}\right) \approx 4733$ would result in a huge $O\left(a^{4}\right)$ coefficient $d_{4}^{L}+h_{4}^{L}=16110$.

Since $\Delta^{L+T}$ has a larger weight on the total contribution to $\delta_{u s}^{(2)}$, the final combination of the transverse and longitudinal pieces has a better behaviour

$$
\begin{aligned}
\Delta[ & \left.a\left(M_{\tau}^{2}\right)\right]=1+\frac{16}{3} a\left(M_{\tau}^{2}\right)+\left(\frac{10775}{144}-\frac{17}{12} \pi^{2}+\frac{1}{9} \zeta_{3}-\frac{130}{9} \zeta_{5}\right) a\left(M_{\tau}^{2}\right)^{2} \\
& +\left(\frac{3}{4} d_{3}^{L+T}+\frac{14050201}{20736}-\frac{5275}{144} \pi^{2}-\frac{\pi^{4}}{144}-\frac{47401}{288} \zeta_{3}+\frac{13195}{432} \zeta_{5}\right) a\left(M_{\tau}^{2}\right)^{3} \\
& +\cdots \\
& =1+5.333 a\left(M_{\tau}^{2}\right)+46.000 a\left(M_{\tau}^{2}\right)^{2}+\left(149.185+\frac{3}{4} d_{3}^{L+T}\right) a\left(M_{\tau}^{2}\right)^{3}+\cdots
\end{aligned}
$$

Nevertheless, the convergence of this perturbative series is very poor for the range of the strong coupling relevant in $\tau$ decays, $a\left(M_{\tau}^{2}\right) \sim 0.11$. With $d_{3}^{L+T} \sim$ $d_{2}^{L+T}\left(d_{2}^{L+T} / d_{1}^{L+T}\right) \approx 317$, the $O\left(a^{3}\right)$ correction would be of the same size as the $O(a)$ and $O\left(a^{2}\right)$ contributions.

\section{Resummation of Running Effects along the Integration Contour}

At the moment, we can do very little about the apparent growth of the $d_{n}^{J}$ coefficients, specially for $J=L$. We clearly need a deeper understanding of the perturbative $\left.D_{u s}^{J}(s)\right|_{D=2}$ expansions. However, we can try to control better the large contributions contained in the $h_{n}^{J}$ factors.

The integration along the circle $x=e^{i \phi}$ gives rise to a long running of the quark mass and the QCD coupling. The expansion of $m^{2}\left(-\xi^{2} M_{\tau}^{2} x\right) a^{n}\left(-\xi^{2} M_{\tau}^{2} x\right)$ in powers of $a_{\xi}$ generates imaginary logarithms $\log ^{n}(-x)=i^{n}(\phi-\pi)^{n}$, which are large in some parts of the integration range. The radius of convergence of such expansion is actually quite small [4]. However, there is no need to perform this ill-defined power expansion.

Using in Eqs. (23) and (24) the exact solution for $m(-s)$ and $a(-s)$ obtained from the renormalization group equations, the $B_{J}^{(n)}\left(a_{\xi}\right)$ integrals can be calculated to all orders in $\alpha_{s}$, apart from the unknown $\beta_{n>4}$ and $\gamma_{n>4}$ contributions, which are likely to be small. Thus, a more appropriate approach is to directly use the expansions (22), in terms of the original $\tilde{d}_{n}^{J}$ coefficients, and to fully keep the known four-loop information on the functions $B_{J}^{(n)}\left(a_{\xi}\right)$.

Tables 1 and 2 show the exact results for $B_{L+T}^{(n)}(a)$ and $B_{L}^{(n)}(a)(n=0,1,2,3)$ with $\xi=1$ obtained at different orders in the $\beta$ and $\gamma$ expansions, together with

${ }^{a}$ The $O\left(a^{2}\right)$ correction agrees with the numerical result recently reported in Ref. [29], which is larger than the value originally quoted in Ref. 16]. This larger $O\left(a^{2}\right)$ correction has been also confirmed by K. Chetyrkin and A. Kwiatkowski [30]. 
the final values of $\Delta^{J}[a]$, for $a=0.1(\xi=1)$. For comparison the numbers coming from the truncated perturbative expressions at $O\left(a^{3}\right)$ are also given.

Table 1: Exact results for $B_{L+T}^{(n)}(a) \quad(n=0,1,2,3)$ obtained at the $\mathrm{k}$-loop $(k=$ $1,2,3,4)$ approximation $\left(\beta_{j>k}=\gamma_{j>k}=0\right)$, together with the final value of $\Delta^{L+T}[a]=\sum_{n=0}^{2} d_{n}^{L+T} B_{L+T}^{(n)}(a)$, for $a=0.1$ and $\xi=1$. For comparison the numbers coming from the truncated expressions at $O\left(a^{3}\right)$ are also given.

\begin{tabular}{|c|cccc|c|}
\hline Loops & $B_{L+T}^{(0)}(a)$ & $B_{L+T}^{(1)}(a)$ & $B_{L+T}^{(2)}(a)$ & \multicolumn{1}{c|}{$B_{L+T}^{(3)}(a)$} & $\Delta^{L+T}[a]$ \\
\hline 1 & 0.89032 & 0.06965 & 0.00452 & 0.000186 & 1.360 \\
2 & 0.81719 & 0.05666 & 0.00278 & -0.000008 & 1.166 \\
3 & 0.79143 & 0.05296 & 0.00236 & -0.000048 & 1.108 \\
4 & 0.78237 & 0.05168 & 0.00222 & -0.000060 & 1.089 \\
\hline$O\left(a^{3}\right)$ & 0.79363 & 0.06473 & 0.00892 & 0.001000 & 1.405 \\
\hline
\end{tabular}

Table 2: Exact results for $B_{L}^{(n)}(a) \quad(n=0,1,2,3)$ obtained at the $\mathrm{k}$-loop $(k=$ $1,2,3,4)$ approximation $\left(\beta_{j>k}=\gamma_{j>k}=0\right)$, together with the final value of $\Delta^{L}[a]=\sum_{n=0}^{3} d_{n}^{L} B_{L}^{(n)}(a)$, for $a=0.1$ and $\xi=1$. For comparison the numbers coming from the truncated expressions at $O\left(a^{3}\right)$ are also given.

\begin{tabular}{|c|cccc|c|}
\hline Loops & $B_{L}^{(0)}(a)$ & $B_{L}^{(1)}(a)$ & $B_{L}^{(2)}(a)$ & $B_{L}^{(3)}(a)$ & $\Delta^{L}[a]$ \\
\hline 1 & 1.39908 & 0.18473 & 0.02255 & 0.002588 & 4.686 \\
2 & 1.54013 & 0.20247 & 0.02421 & 0.002692 & 5.052 \\
3 & 1.57853 & 0.20617 & 0.02444 & 0.002690 & 5.120 \\
4 & 1.58910 & 0.20706 & 0.02446 & 0.002681 & 5.133 \\
\hline$O\left(a^{3}\right)$ & 1.64446 & 0.21894 & 0.02192 & 0.001000 & 4.356 \\
\hline
\end{tabular}

These numerical results show a reasonable convergence of the $B_{J}^{(n)}(a)$ integrals, as higher-order $\beta_{k}$ and $\gamma_{k}$ contributions are taken into account. Increasing the number of loops one gets a small decrease (increase) of the transverse (longitudinal) contribution. It is also clear that the truncated $O\left(a^{3}\right)$ expressions overestimate (underestimate) $\Delta^{L+T}\left(\Delta^{L}\right)$. Taking the full four-loop information into account, we get the following perturbative behaviour:

$$
\begin{gathered}
\Delta^{L+T}[0.1]=0.7824+0.2239+0.0823-0.0000601 d_{3}^{L+T}+\cdots \\
\Delta^{L}[0.1]=1.5891+1.1733+1.1214+1.2489+\cdots
\end{gathered}
$$

The $L+T$ series converges very well. Owing to the negative running contributions the $\Delta^{L+T}[a]$ series behaves better than the original perturbative expansion of $\left.D_{u s}^{L+T}(s)\right|_{D=2}$. Unfortunately, the longitudinal series is much more problematic. 
The bad perturbative behaviour of $\left.D_{u s}^{L}(s)\right|_{D=2}$ gets reinforced by the running effects, giving rise to a badly defined series.

The combined final expansion,

$$
\Delta[0.1]=0.9840+0.4613+0.3421+\left(0.3122-0.000045 d_{3}^{L+T}\right)+\cdots
$$

looks acceptable for the firsts terms because $\Delta^{L+T}$ is weighted by a larger factor. In fact, this series behaves better than the one in Eq. (39), obtained with the usual perturbative truncation of the contour integrals. Nevertheless, after the third term the series appears to be dominated by the longitudinal contribution, and the bad perturbative behaviour becomes again manifest.

Using the full four-loop result we have certainly gained in convergence for the $\Delta^{L+T}$ series [compare the fourth term in the series (37) and (40) for $a=0.1$ ], which is otherwise the one we don't know the $O\left(a^{3}\right)$ coefficient. We can take advantage that the $O\left(a^{3}\right)$ correction to $\Delta[a]$ is almost completely given by the known $\Delta^{L}$ contribution. Using $d_{3}^{L+T} \sim d_{2}^{L+T}\left(d_{2}^{L+T} / d_{1}^{L+T}\right) \approx 317$, the fourth term in (42) becomes 0.298 , i.e. a $5 \%$ reduction only. Taking the size of the $O\left(a^{3}\right)$ contribution to $\Delta^{L}$ as an educated estimate of the perturbative uncertainty, we finally get

$$
\Delta[0.1]=2.1 \pm 0.3
$$

\section{Renormalization-Scale Dependence}

The expansion (22) depends order by order on $\xi$ and this dependence cancels out only when we sum the infinite series. In practice, we only know a few first terms of the series (three for $\Delta^{L+T}[a]$ and four for $\Delta^{L}[a]$ ); so we should worry how much the predictions depend on our previous choice $\xi=1$. Obviously, $\xi$ should be close to one in order to avoid large logarithms; but variations within a reasonable range, let us say from 0.75 to 2 , should not affect too much the final results. Smaller values of $\xi$ would put the QCD coupling in the non-perturbative regime and are therefore not acceptable.

Figures 1, 2 and 3 show the sensitivity to the selection of renormalization scale of the final predictions for $\Delta^{L+T}, \Delta^{L}$, and $\Delta$, respectively, for $a\left(M_{\tau}^{2}\right)=0.1$.

The behaviour of $\Delta^{L+T}$ is quite good. The predicted value remains very stable in the whole range $\xi \in[0.75,2]$, showing that the perturbative series is very reliable. Below $\xi \sim 1 / 2$, the perturbative expansion breaks down, as expected, because the coupling $a_{\xi}$ is already outside the radius of convergence of the series.

The longitudinal series, on the other side, has a quite wild dependence on the renormalization scale. Changing $\xi$ from 1 to 2 , amounts to a reduction of $\Delta^{L}$ of about $65 \%$. Thus, the theoretical uncertainty is very large in this case.

The $\xi$ dependence of the complete expansion $\Delta$, reflects obviously the behaviour of its two components. The larger weight of $\Delta^{L+T}$ keeps the result still acceptable, within the range of $\xi$ considered, but the sizeable $\Delta^{L}$ contribution 
spoils the stability and generates a monotonic decrease of the prediction for increasing values of $\xi$. Taking this variation into account, the theoretical error in Eq. (43) should be increased to about 0.6, i.e. a $30 \%$ uncertainty in the final prediction.

\section{Discussion}

The bad perturbative behaviour of the longitudinal contribution does not allow to make an accurate determination of the strange quark mass from $R_{\tau, S}$. Nevertheless, taking

$$
\Delta[0.1]=2.1 \pm 0.6
$$

$m_{s}\left(M_{\tau}^{2}\right)$ could be still obtained with a theoretical uncertainty of about $15 \%$, which is not so bad.

Notice that it is the phase-space integration of the original correlation functions the responsible for the different behaviour of the longitudinal and transverse components. Therefore, the perturbative convergence could probably be improved through an appropriate use of weight factors in Eqs. (5) and (8). This requires an accurate measurement of the final hadrons mass distribution in the $\tau$ decay, which so far has only been performed for the dominant Cabibbo-allowed modes [31]. The measurement of $R_{u s}(s)$ could be feasible at the forthcoming flavour factories, where a very good kaon identification is foreseen.

From the theoretical point of view, the analysis of weighted moments of the final hadrons mass distribution proceeds in a completely analogous way [32]. A detailed study will be presented in a forthcoming publication.

\section{Acknowledgements}

We would like to thank Kostja Chetyrkin for informing us about the existence of a misprint in Ref. [16]. We have benefit from many discussions (and program exchanges) with Matthias Jamin and Arcadi Santamaría, concerning the numerical implementation of running effects at higher orders. Useful discussions with Michel Davier, Andreas Höcker and Eduardo de Rafael are also acknowledged. This work has been supported in part by the European Union TMR Network EURODAPHNE - Contract No. ERBFMRX-CT98-0169 (DG 12 - MIHT) and by CICYT, Spain, under Grants No. AEN-96/1672 and AEN-96/1718. 


\section{References}

[1] E. Braaten, Phys. Rev. Lett. 60 (1988) 1606; Phys. Rev. D39 (1989) 1458.

[2] S. Narison and A. Pich, Phys. Lett. B211 (1988) 183.

[3] E. Braaten, S. Narison, and A. Pich, Nucl. Phys. B373 (1992) 581.

[4] F. Le Diberder and A. Pich, Phys. Lett. B286 (1992) 147.

[5] A. Pich, QCD Tests from Tau Decays, Proc. of the $20^{\text {th }}$ Johns Hopkins Workshop on Current Problems in Particle Theory - Non-Perturbative Particle Theory and Experimental Tests - (Heidelberg, June 1996), eds. M. Jamin et al (World Scientific, Singapore, 1997), p. 247.

[6] S. Chen, Nucl. Phys. B (Proc. Suppl.) 64 (1998) -.

[7] M. Davier, Nucl. Phys. B (Proc. Suppl.) 55C (1997) 395.

[8] C. Becchi et al, Z. Phys. C8 (1981) 335.

[9] C.A. Domíguez and E. de Rafael, Ann. Phys., NY 174 (1987) 372.

[10] M. Jamin and M. Münz, Z. Phys. C66 (1995) 633.

[11] J. Bijnens, J. Prades, and E. de Rafael, Phys. Lett. B348 (1995) 226.

[12] M. Jamin, Nucl. Phys. B (Proc. Suppl.) 64 (1998) 250.

[13] J. Prades, Nucl. Phys. B (Proc. Suppl.) 64 (1998) 253.

[14] K.G. Chetyrkin, Phys. Lett. B390 (1997) 309.

[15] P.M. Stevenson, Phys. Rev. D23 (1981) 2916.

[16] K.G. Chetyrkin and A. Kwiatkowski, Z. Phys. C59 (1993) 525.

[17] K.G. Chetyrkin and J.H. Kühn, Phys. Lett. B406 (1997) 102.

[18] M.A. Shifman, A.L. Vainshtein, and V.I. Zakharov, Nucl. Phys. B147 (1979) 385; 448; 519.

[19] W.J. Marciano and A. Sirlin, Phys. Rev. Lett. 61 (1988) 1815; 56 (1986) 22.

[20] E. Braaten and C.S. Li, Phys. Rev. D42 (1990) 3888.

[21] T. van Ritbergen, J.A.M. Vermaseren, and S.A. Larin, Phys. Lett. B400 (1997) 379; B405 (1997) 327. 
[22] K.G. Chetyrkin, B.A. Kniehl, and M. Steinhauser, Phys. Rev. Lett. 79 (1997) 2184.

[23] K.G. Chetyrkin, Phys. Lett. B404 (1997) 161.

[24] L.R. Surguladze, Sov. J. Nucl. Phys. 50 (1989) 372.

[25] S.G. Gosrishny et al, Mod. Phys. Lett. A5 (1990) 2703.

[26] S.G. Gosrishny et al, Phys. Rev. D43 (1991) 1633.

[27] S.G. Gorishny, A.L. Kataev, and S.L. Larin, Nuovo Cimento 92 ( 1986) 119.

[28] W. Bernreuther and W. Wetzel, Z. Phys. C11 (1981) 113.

[29] K. Maltman, Problems with Extracting $m_{s}$ from Flavor Breaking in Hadronic $\tau$ Decays, York preprint YU-PP-K/M-98-1, hep-ph/9804298.

[30] K.G. Chetyrkin, private communication.

[31] R. Barate et al (ALEPH Coll.), Measurement of the Spectral Functions of Axial-Vector Hadronic $\tau$ Decays and Determination of $\alpha_{s}\left(M_{\tau}^{2}\right)$, preprint CERN-EP-98-012

[32] F. Le Diberder and A. Pich, Phys. Lett. B289 (1992) 165. 


\section{Figure Captions}

- Figure 1.- Variation of $\Delta_{L+T}[0.1]$ with the renormalization-scale factor $\xi$, to four loops.

- Figure 2.- Variation of $\Delta_{L}[0.1]$ with the renormalization-scale factor $\xi$, to four loops.

- Figure 3.- Variation of $\Delta[0.1]$ with the renormalization-scale factor $\xi$, to four loops. 


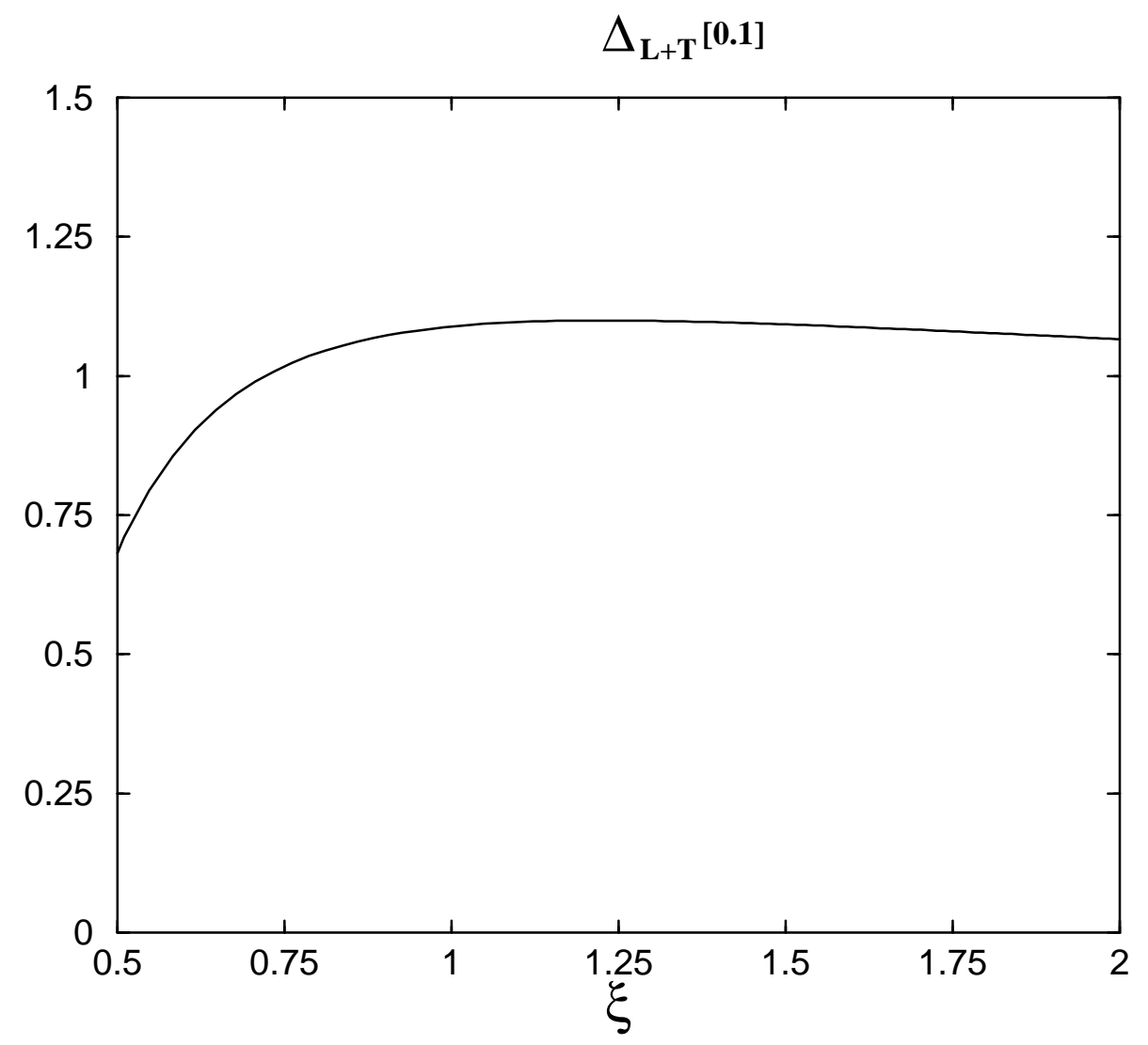

Figure 1: Variation of $\Delta_{L+T}[0.1]$ with the renormalization-scale factor $\xi$, to four loops. 


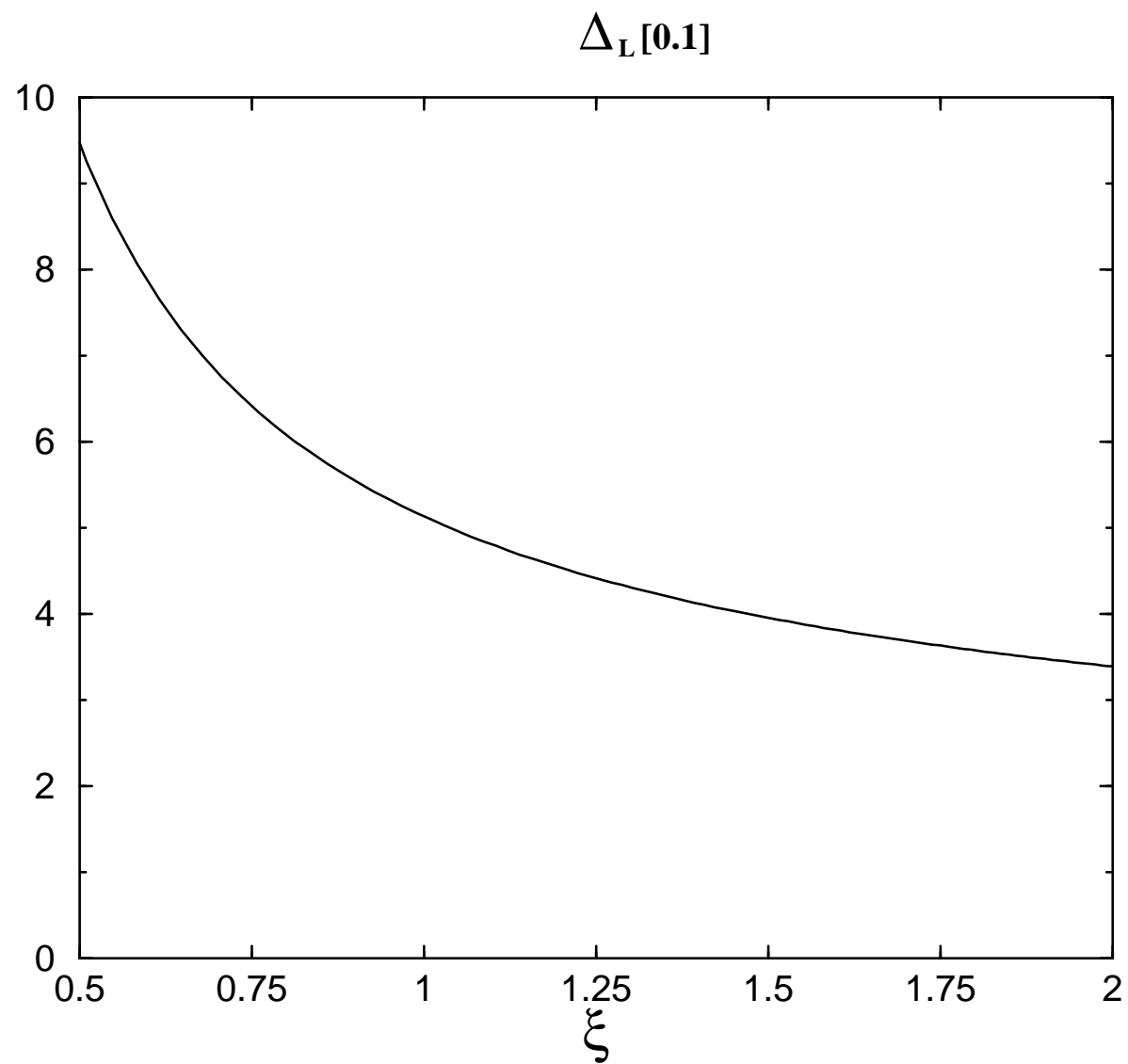

Figure 2: Variation of $\Delta_{L}[0.1]$ with the renormalization-scale factor $\xi$, to four loops. 


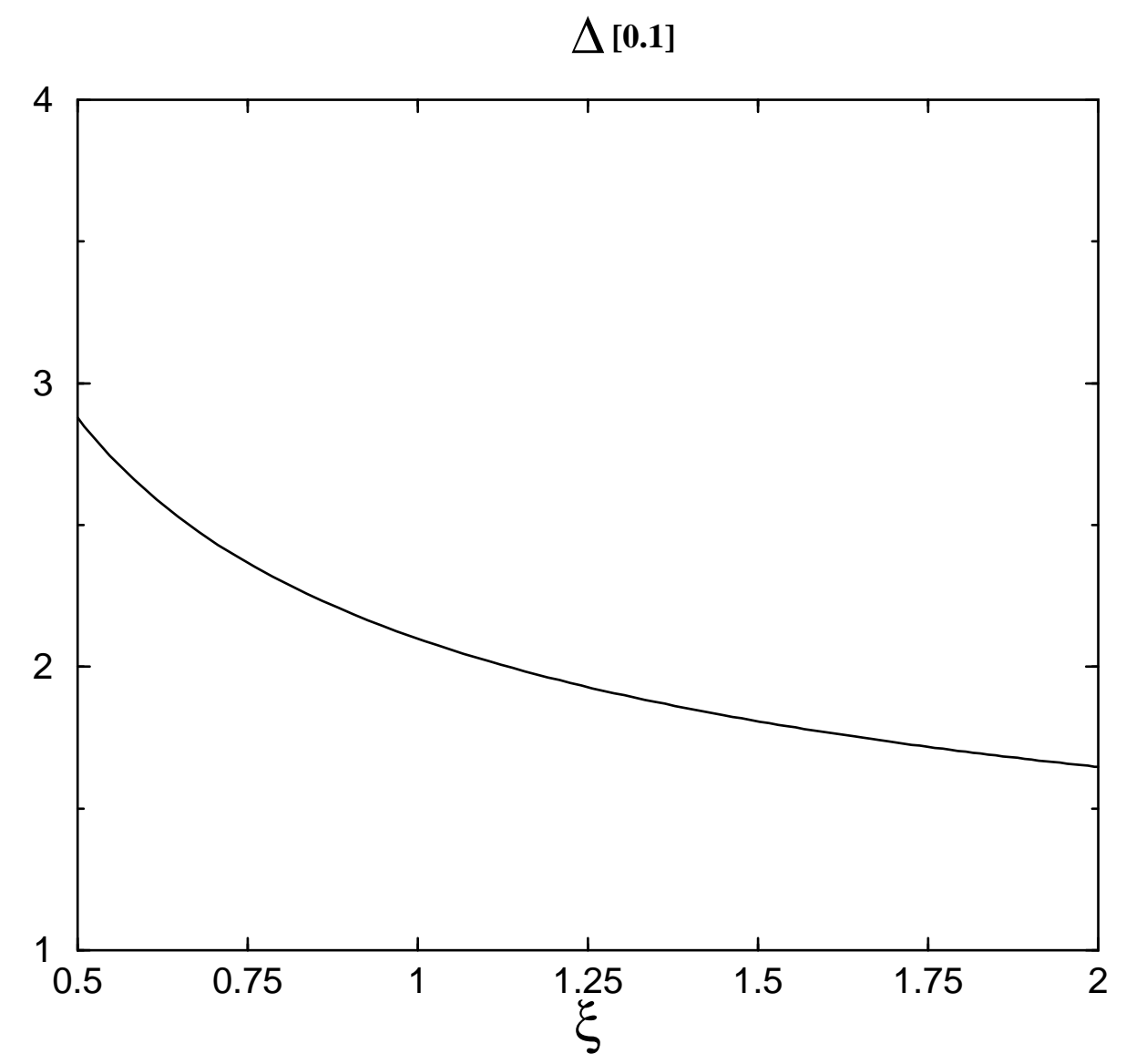

Figure 3: Variation of $\Delta[0.1]$ with the renormalization-scale factor $\xi$, to four loops. 